\title{
Substrate area confinement is a key determinant of cell velocity in collective migration
}

\author{
Danahe Mohammed $^{1}$, Guillaume Charras ${ }^{2,3}$, Eléonore Vercruysse ${ }^{1}$, Marie Versaevel ${ }^{1}$, \\ Joséphine Lantoine $^{1}$, Laura Alaimo ${ }^{1}$, Céline Bruyère ${ }^{1}$, Marine Luciano ${ }^{1}$, Karine Glinel ${ }^{4}$ \\ Geoffrey Delhaye ${ }^{5}$, Olivier Théodoly ${ }^{5}$, and Sylvain Gabriele ${ }^{{ }^{*}}$
}

\begin{abstract}
Collective cell migration is fundamental throughout development, wound healing and in many diseases. Although much effort has focused on cell-cell junctions, a role for physical confinement in collective cell migration remains unclear. Here we used adhesive microstripes of varying widths to mimic the spatial confinement experienced by follower cells within epithelial tissues. Our results reveal that the substrate area confinement is sufficient to modulate the three-dimensional (3D) cellular morphology without the need for intercellular adhesive cues. Our findings show a direct correlation between the migration velocity of confined cells and their cell-substrate adhesive area. Closer examination revealed that adhesive area confinement reduces lamellipodial protrusive forces, decreases the number of focal complexes at the leading edge and prevents the maturation of focal adhesions at the trailing edge, leading together to less effective
\end{abstract}

\footnotetext{
${ }^{1}$ Mechanobiology \& Soft Matter group, Laboratoire Interfaces et Fluides Complexes, Research Institute for Biosciences, University of Mons, Place du Parc, 20 B-7000 Mons, Belgium

${ }^{2}$ London Centre for Nanotechnology, University College London, 17-19 Gordon Street, London WC1H OAH, $U K$

${ }^{3}$ Department of Cell and Developmental Biology, University College London, London WC1E 6BT, UK.

${ }^{4}$ Institute of Condensed Matter \& Nanosciences, Bio \& Soft Matter division, Université Catholique de Louvain, Croix du Sud, box L7.04.02, B-1348 Louvain-la-Neuve, Belgium

${ }^{5}$ Adhésion \& Inflammation, INSERM U1067-CNRS UMR7333, Université Aix-Marseille, Assistance PubliqueHôpitaux de Marseille, Case 937, 163 Avenue de Luminy, F-13009 Marseille, France

*Correspondence should be addressed to S.G. (email: sylvain.gabriele@umons.ac.be)
} 
forward propelling forces. The release of follower confinement required for the emergence of leader cells is associated with a three-fold increase in contractile stress and a ten-fold increase in protrusive forces, providing together a sufficient stress to generate highly motile mesenchymal cells. These findings demonstrate that epithelial confinement alone can induce follower-like behaviours and identify substrate adhesive area confinement as a key determinant of cell velocity in collective migration.

Cell migration is a fundamental biological process in which individual cells orchestrate their movement by interacting with each other and with their environment. In two-dimensional (2D) environments, cells migrate as large epithelial sheets during many biological processes, such as tissue morphogenesis ${ }^{1}$, wound closure ${ }^{2}$, embryonic development ${ }^{2}$ or tumor metastasis ${ }^{4}$. Migration of epithelium-derived cells consists of basic sequential events occurring in a cyclic fashion ${ }^{5}$. This migration mode depends on the formation of adhesion sites, actin-driven protrusion generation, and actomyosin contractility that together create stress within the cell ${ }^{6}$. Migration of 2D epithelial sheets occurs under a broad range of external constraints that induce the appearance of highly motile mesenchymal cells at the leading front that guide follower cells ${ }^{7}$. Among the different environmental constraints, recent evidence suggests that a 2D confinement may modulate the transition between collective and single cell migration ${ }^{8}$ by inducing a restriction of cell-cell contacts, leading to the dispersion of cells from a collective sheet ${ }^{9}$. Eventually, leader cells tend to escape and migrate separately leading to the progressive dissociation of the cell cluster. Behind the leader cells, cell compaction impairs the formation of a classical leading edge, suggesting that physical confinement affects not only cell morphology but also the core biological mechanisms driving migration of follower cells.

Cadherin-mediated interactions between neighbouring cells contribute to the polarization of migrating cell groups and it has been shown that impairing cadherin function dramatically alters collective migration dynamics ${ }^{7}$. Although much effort has focused on the importance of cell-cell junctions ${ }^{10}$, the effect of physical 2D confinement on the migration of 
single cells or epithelial cell sheets has been recognized but not yet elucidated. This is surprising because cells in epithelial sheets are often naturally compacted and have access to smaller adhesion area with the substrate. Recent reports suggest that density mediated contact inhibition of locomotion ${ }^{11,12}$ can be described using an analogy between a cell monolayer and a bidimensional "jammed" colloidal system ${ }^{13,14}$, emphasizing the role of spatial confinement as a control parameter for wound healing and epithelial cell migration. However, our current understanding of even the most basic aspects of epithelial cell migration under physical confinement remains poor.

Here we study how the restriction of the cell-substrate contact area can modulate epithelial migration. We used adhesive micropatterned stripes to examine the motion of confined fish epithelial keratocytes, a classic model system to investigate single cell motility ${ }^{15-17}$. A wide range of physical 2D confinements, similar to those encountered physiologically by cells when migrating collectively, was imposed by varying the width of adhesive microstripes. Furthermore, we use keratocyte explants as an efficient primary model of collective cell migration. The modulation of the available adhesive area in confined environments emerges as a key physical parameter that regulates cell morphology, driving forces and migrating velocity in collective cell migration.

\section{Followers are elongated, laterally confined and slower}

When scales are removed from a fish and deposited onto a substrate homogeneously coated with fibronectin $(\mathrm{FN})$, this leads to the formation of a $2 \mathrm{D}$ migrating epithelial tissue that spreads onto the substrate replicating wound healing (Fig. 1A and Supplementary Movie 1). As shown in Fig. 1A, there are clear differences between follower and leader cells after $14 \mathrm{~h}$ in explant culture. Leader cells were polarized and elongated along the direction perpendicular to the direction of migration. They possessed a broad actin-rich lamellipodium, whereas their microtubules were wrapped around the nucleus in the cell body. In contrast, followers were mostly elongated along the migration direction, exhibited a prominent cortical actin network and a microtubule network that extended throughout the cell. The mean aspect ratio, as denoted by the ratio of the cell axis perpendicular to the migration direction to the 
cell axis parallel to the migration direction, was $0.44 \pm 0.11$ for confined cells $(n=42)$ and $1.58 \pm 0.24$ for leaders $(n=47)$, suggesting important symmetry breaking dependent on cell position (Fig. 1B). Furthermore, followers have a smaller mean width $(11.1 \pm 2.3 \mu \mathrm{m}, \mathrm{n}=52$,

Supplementary Fig. 1A) than leaders (22.5 $\pm 2.6 \mu \mathrm{m}, \mathrm{n}=49$, Supplementary Fig. 1B). As shown in Figs. 1C-D, followers had a smaller projected area $\left(190 \pm 49 \mu \mathrm{m}^{2}, \mathrm{n}=42\right)$ and were slower $(0.03 \pm 0.05 \mu \mathrm{m} / \mathrm{s}, \mathrm{n}=40)$ than leaders $\left(475 \pm 20 \mu \mathrm{m}^{2}, \mathrm{n}=47\right.$ and $\left.0.05 \pm 0.08 \mu \mathrm{m} / \mathrm{s}, \mathrm{n}=44\right)$.

Together, these results raise the possibility that spatial confinement experienced by epithelial cells might control their morphology and migration velocity.

\section{Confinement regulates cell morphologies and velocities}

To address this question, we examined the motile behaviour of individual keratocytes migrating on FN microstripes of well-defined widths ranging from 5 to $20 \mu \mathrm{m}$ (Fig 1E) to reproduce the range of lateral 2D confinements encountered within epithelial tissues (Supplementary Fig. 1). As shown in Fig. 1F and Supplementary Movie 2, individual keratocytes migrating on narrow microstripes modified their typical fan-shaped morphology and increased their long axis to elongate in the direction of migration. We found that the cell aspect ratio decreased linearly with the microstripe width (Fig. $2 \mathrm{~A}, \mathrm{R}^{2}=0.948$ ). Interestingly, spatial confinement also modulated the fraction of stationary cells that decreased from $0.98 \pm 0.01$ on the narrowest microstripe to $0.07 \pm 0.01$ on the largest one ( $n=48$, Fig. $2 B)$. The linear adaptation of the cell projected area to the lateral confinement imposed by the microstripe width was mainly driven by a reorganization of the lamellipodium (Fig. 2C).

Time-lapse recording of individual keratocytes migrating on microstripes indicated that cells moved with a constant velocity that can be modulated by the microstripe width, as observed by slope changes (Fig. 2D). Our results showed that the evolution of migration velocity with the micropattern width was described by two distinct domains with a sharp transition around $10 \mu \mathrm{m}$ (Fig. 2E), corresponding to a spreading area of $\sim 190 \mu \mathrm{m}^{2}$ (Fig. 2F). For widths below $10 \mu \mathrm{m}$, keratocytes migrated very slowly $(\sim 0.02 \mu \mathrm{m} / \mathrm{s})$, whereas above 10 $\mu \mathrm{m}$ the migration velocity increased linearly with microstripe width to reach $\sim 0.35 \mu \mathrm{m} / \mathrm{s}$ on $20 \mu \mathrm{m}$ wide microstripes, similar to the velocity observed in unconfined cells ${ }^{18}$. To confirm 
these observations, we next designed a pattern composed of five interconnected microstripes of increasing widths: 5, 9, 13, 17 and $21 \mu \mathrm{m}$ (Figs. $2 \mathrm{G}$ and H). The migration of individual confined cells $(n=13)$ on interconnected microstripes was tracked by time-lapse microscopy during several forward and backward travels (Supplementary Movie 3). The small difference in width between two successive segments (i.e. $4 \mu \mathrm{m}$ ) ensured a smooth transition in velocity. As shown in Fig. 2I, the velocity increased from narrow to large microstripes (forth trajectory), then cells turned back at the end of the larger microstripe and the migrating distance over time decreased from large to narrow microstripes (back trajectory). The overlay of two successive forth trajectories of the same cell indicated a robust and reproducible adaptation of migrating behaviour to physical confinement (Fig. 2J). The instantaneous velocity on forth and back travels (Fig. 2K) was obtained by differentiating the sigmoidal fit of the migrating distance versus time (Fig. 2I). Next, we grouped follower cells in bins centered on the widths of the microstripes. The average area of followers within each bin was calculated and plotted as a function of the area of cells confined on microstripes with a width equal to the bin of interest (Supplementary Fig. 3). We found a linear correlation (slope = $0.99 \pm 0.04$ and $R^{2}=0.9015, n=93$ ) between the projected area of followers and the projected area of confined cells for different microstripe widths. Confined cells cannot maintain their migrating phenotype on microstripes narrower than $11 \mu \mathrm{m}$, which is just below the mean width of followers $(\mathrm{w}=12.1 \pm 2.2 \mu \mathrm{m}, \mathrm{n}=52$, Supplementary Fig. 1$)$.

Together, these results suggest that lateral confinement, which is the dominant form of confinement experienced by follower cells, regulates morphologies and velocities of migrating cells.

\section{D physical confinement modulates 3D cell morphology}

We next investigated the three-dimensional (3D) cytoskeletal organization (F-actin, microtubules and DNA) of confined keratocytes by confocal microscopy. The leading edge of fan-shaped cells was composed of a flat lamellipodium enriched with F-actin ${ }^{19}$, whereas the trailing edge was characterized by a spherical cell body containing a dense network of microtubules wrapped around the nucleus (Fig. 3A). In contrast, confined keratocytes 
displayed an elongated morphology with a thicker leading edge and a large accumulation of F-actin on both cell sides. Surprisingly, our results revealed that microtubules extended to the leading edge of confined cells (Fig. 3B), as observed in followers during collective migration (Fig. 3C). These findings suggest that physical confinement alone is sufficient to reorganize actin and microtubule networks without the need for intercellular adhesive cues. Our results showed that isolated cells exhibited a random orientation of microtubules (Supplementary Fig. 4A-C), whereas confined cells (Supplementary Fig. 4D-F) and followers (Supplementary Fig. 4G-J) were characterized by a network of microtubules oriented parallel to the axis of migration. In addition, cells confined on microstripes showed similar values of coverage area of microtubules to overall cell area $(45.5 \pm 5.9 \%)$ than followers $(40.6 \pm 8.5 \%)$, whereas unconfined cells exhibited lower values $(18.3 \pm 1.2 \%)$. Together, these results strengthen the morphological similarities between cells confined on narrow adhesive microstripes and follower cells compacted within epithelial tissues (Supplementary Fig. 4K).

We acquired cell height profiles along the migration axis of glutaraldehyde fixed isolated and confined keratocytes using atomic force microscopy (Fig. 3D and Supplementary Fig. 5). The rounded cell body of unconfined cells formed an angle of $\sim 78 \pm 4^{\circ}(n=11)$ with the flat leading edge (Fig. 3E), indicating an abrupt transition between leading and trailing edges. In contrast, confined keratocytes exhibited a more elongated morphology and a lower transition angle $\left(\sim 42 \pm 7^{\circ}, n=13\right)$, suggesting a smooth transition between both subcompartments. We then quantified the mean height of both cell microcompartments by acquiring profiles perpendicular to the cell migration axis for keratocytes on 20,10 and $5 \mu \mathrm{m}$ wide microstripes (Fig. 3G). Surprisingly, our results showed that the cell body flattened significantly on narrow stripes ( $5 \mu \mathrm{m}$ wide) down to $1.3 \pm 0.2 \mu \mathrm{m}$ (Fig. $3 \mathrm{H}, \mathrm{n}=13$ ), corresponding to a decrease of $\sim 59 \%$ in height for confined cells. Conversely, we observed a significant thickening of the leading edge that reached $580 \pm 50 \mathrm{~nm}$ on $5 \mu \mathrm{m}$ wide microstripes (Fig. 3I, $\mathrm{n}=11$ ), corresponding to a $\sim 285 \%$ increase of the thickness. On $20 \mu \mathrm{m}$ wide microstripes, keratocytes adopted a fan-shaped morphology characterized by a mean volume of the cell body about 8 times higher than the leading edge volume. Despite large 
morphological modifications of cell body (Fig. 3J) and leading edge (Fig. 3K) in confined cells, the total cell volume remained constant (Fig. 3L).

Together, these results show that $2 \mathrm{D}$ confinement induces significant $3 \mathrm{D}$ modifications of cell morphology with a redistribution of cytoskeletal components and large variations of volume between leading and trailing edges, while keeping the total cellular volume constant.

\section{Thicker lamellipodia exert less protrusive forces}

Based on the central role of F-actin assembly and disassembly in keratocyte motility ${ }^{20}$, we next hypothesized that the slower velocity of keratocytes confined on microstripes might be a consequence of the leading edge thickening, which may reduce the efficiency of polymerization driven membrane protrusion. To test this experimentally, we measured the lamellipodial protrusive forces by placing an AFM cantilever of calibrated spring constant perpendicular to the surface in front of the leading edge, as introduced previously ${ }^{21}$. The vertical position of the cantilever was measured optically over time (Figs. 4A-B and Supplementary Movie 4). When the lamellipodium was stalled at higher forces, the velocity of the lamellipodium decreased eventually to zero. The first derivative of the deflection-time curves was used to obtain the cantilever speed versus time (Fig. 4C) and the lamellipodial protrusive force was determined from the mean stall force, when the cantilever movement stopped. Our results showed that protrusive forces exerted by the leading edge increased with cell velocity (Fig. 4D), which was itself controlled by the microstripe width. As a consequence, protrusive forces increased linearly with microstripe width (Fig. 4E). Assuming a mean force of $\sim 8 \mathrm{pN}$ generated per actin filament ${ }^{22}$ and knowing the height of the lamellipodium (Fig. 2I), we estimated that only $\sim 25$ actin filaments pushed on a contact area of $3.5 \mu \mathrm{m}^{2}$ in highly confined cells. This corresponded to $\sim 7$ filaments $/ \mu \mathrm{m}^{2}$. In contrast, $\sim 300$ actin filaments pushed on a contact area of $2.5 \mu \mathrm{m}^{2}$ in unconfined cells, corresponding to $\sim 120$ filaments $/ \mu \mathrm{m}^{2}$. The pressure exerted by actin filaments was therefore significantly modulated by the width of the microstripe (Fig. 4F). 
Together, these results revealed that physical confinement imposed by narrow microstripes leads to less effective protrusive force generation in thicker leading edges that reduce the migration velocity.

\section{Escaping tissues requires the actin cytoskeleton remodeling}

Next we sought to understand the mechanisms allowing leader cells to escape from the tissue. The escape process from primary epithelial sheets can be described as a succession of three states: (i) confined, (ii) transition and (iii) unconfined (Fig. 5A and Supplementary Movie 5). We reproduced this process using a new micropattern geometry composed of a linear microstripe of $12 \mu \mathrm{m}$ wide connected to a disk of $50 \mu \mathrm{m}$ in diameter (Fig. 5B and Supplementary Movie 6). As soon as the cell extremity reached the circular zone, the leading edge tended to adopt a fan shape, whereas the cell body was still confined on the narrow microstripe.

We found that the cell projected area increased for cells escaping a tissue ( $75 \%$, Fig. 5D) and a microstripe ( 71\%, Fig. 5E). Spatiotemporal kymograph analysis of the escape process indicated that the three successive steps were associated with unsynchronized velocity changes in the leading and trailing edges (Fig. 5F). Indeed, confined cells entering the circular disk stopped the progression of their leading edge (Fig. 5G) until their trailing edge had exited the microstripe. At this transition stage, keratocytes reorganized their actin cytoskeleton to extend their leading edge on the circular disk. Using Sir-actin to label F-actin in live cells (Fig 5I and Supplementary Movie 7), we showed the presence of two intense fluorescent signals in confined cells located on their lateral sides and oriented along to the migration axis, as observed in immunostainings (Fig. 3B). Interestingly, the F-actin signal disappeared at the transition between the microstripe and the circular disk (Fig. 5J) and unconfined cells formed a large lamellipodium enriched with F-actin. To get insight into actin reorganization, we

performed additional time-lapse experiments with GFP-UTR keratocytes (Supplementary Fig. 6A and Movie 8). We confirmed an increase of the spreading area during the transition (Supplementary Fig. 6B). During the confined stage (Supplementary Fig. 6C), cells generated large and dynamic protrusive extensions composed of densely packed bundles of actin 
filaments that extended parallel to the microstripe axis (Supplementary Movie 8). Lateral membrane ruffles were highly dynamic (Supplementary Fig. 6D-F) and symmetric with a mean angle of $\sim 31^{\circ}$, corresponding to a velocity of $\sim 0.15 \mu \mathrm{m} / \mathrm{sec}$. Previous reports have shown that membrane ruffles, which form in conditions of low cell-substrate adhesion, lead to a significantly reduced lamellipodium persistence and an inefficient cell migration ${ }^{23}$. Our observations indicated that membrane ruffles were associated with confined migration modes, which were characterized by a low number of focal complexes in the leading edge.

Interestingly, membrane ruffles disappeared during the relaxation stage when a network of actin filament formed at the cell edge, leading to an efficient migration mode characterized by a dynamic actin retrograde flow and higher protrusive forces (Supplementary Fig. 6G-I).

These results reveal that the transition from followers to leaders required a profound remodeling of the actin network to adopt larger spreading areas and faster migration speeds.

\section{Migration velocity is regulated by adhesive area}

One hypothesis to explain how physical confinement affects cell migration is a modification of the spatial distribution of cell-substrate adhesions that may, in turn, affect traction forces exerted on the substrate. To address this issue, we determined the distribution of vinculincontaining adhesions during the escape process with confocal microscopy (Fig. 6A). We observed numerous focal complexes $\left(\leq 1 \mu \mathrm{m}^{2}\right)$ at the leading edge of confined and unconfined cells, whereas mature elongated adhesions were mainly found at the trailing edges of unconfined cells. The number of focal complexes in the leading edge increased with the spreading area leading to a constant density of focal complexes (Fig. 6B). The mean area of focal complexes remained constant, despite large modifications of the leading edge morphology during the escape process (Fig. 6C). We next sought to determine the influence of physical confinement on mature focal adhesions $\left(\geq 3 \mu \mathrm{m}^{2}\right)$ at the cell rear. To compare the individual role of both adhesion types, we normalized the area of focal complexes at the front and mature focal adhesions at the rear by the total cell spreading area. We found that adhesion area in the leading edge increased slightly, whereas focal adhesions at the rear matured significantly (Fig. 6D). Interestingly, the normalization of the speed of confined cells 
migrating on adhesive microstripes of various widths to their total adhesive area demonstrated that cell velocity was directly correlated to adhesive area (Fig. 6E), suggesting that physical confinement regulates cell velocity through cell-substrate adhesions. Taken together, these results disclose a distinctive pattern of adhesions, which is directly correlated with physical confinement and cell migration speed.

We used traction force microscopy (TFM) to quantify the amount of contractile forces exerted on the substrate by keratocytes escaping a confined zone (Fig. 6F). Confined cells exerted two-times less contractile stress than isolated cells, which reached a plateau of total stress around $9 \mathrm{kPa}$ (Fig. 6G). Intense contractile forces that were mostly distributed in the leading edge region of confined cells shifted to the trailing edge during the escape process. Our findings show that the amount of traction forces increased in leading edges as confinement was released, whereas traction forces in trailing edges were significantly higher in unconfined cells (Fig. 6H).

We next investigated the role of cell-cell adhesions by forming "trains" of migrating cells on FN-coated microstripes of $15 \mu \mathrm{m}$ wide (Fig. 6I and J). During its spreading, a nontrypsinized tissue forms multicellular protrusions, known as "fingers", that extend on the microstripes and lead to trains of cells of different lengths. Such collectively migrating trains arise from the native epithelial monolayer which was not subjected to a trypsin treatment to preserve endogenous cell-cell contacts (Fig. 6K and Supplementary Fig. 7). We quantified the projected area and the migrating distance of trains of cells composed of $n=2,3$ and 4 cells during time-lapse experiments (Figs. 6L and M). All trains of cells show a persistent migration (Supplementary Movie 9) and behave as a cohesive unit during the whole experiment (Fig. 6N), independently of the cohort size. Moreover, the spreading area increased linearly with the number of cells in the cohort (Fig. 6O). By normalizing the train velocity by the velocity of single cells confined on the same microstripe (Fig. 6L), we found that the velocity of cell trains ( $n=2,3$ or 4 cells) with mature cell-cell adhesions was not statistically different than isolated cells (Fig. 6P). As shown in Supplementary Fig. 8, followers $(0.119 \pm 0.023)$ and confined cells $(0.115 \pm 0.018)$ exhibited statistically the same amount of normalized vinculin area, whereas leader cells showed larger values $(0.253 \pm$ 
0.029), which were close to those observed for individual cells on fibronectin $(0.239 \pm 0.032)$. Taken together, these results confirm that a physical confinement modulates the adhesiveness of keratocytes and suggest that the establishment of head-to-tail adhesive interactions does not modulate the migrating velocity of confined epithelial cells. This result was confirmed for very large trains of cells $(n=13)$ that exhibited a similar migrating velocity than isolated cells (Fig. 6P).

Cohesiveness was usually associated with a reduced migration speed ${ }^{24}$ and whenever rapid migration occurs physiologically, cells tend to downregulate E-cadherin and dissociate through a complete epithelial-to-mesenchymal transition (EMT). However, in many physiological situations, cells undergo an incomplete EMT, in which E-cadherin adhesion is weakened to enable dynamic flexibility for each individual cell within the group, while keeping a certain degree of cohesiveness ${ }^{25}$. As summarized in Supplementary Fig. 9, our results establish for the first time that the substrate area confinement resulting from a lateral cell compaction is a key mechanism in the modulation of cell velocity in collective migration.

\section{Methods}

Stamp fabrication and microcontact printing. Microstripes with different widths (from 5 to $20 \mu \mathrm{m}$ ) and a relaxation pattern (a microstripe of $12 \mu \mathrm{m}$ wide connected to a disk of $50 \mu \mathrm{m}$ in diameter) are created using a silicon master fabricated by deep reactive-ion etching from a chromium photomask (Toppan Photomask, Corbeil Essonnes, France). The silicon surface was passivated with a fluorosilane (tridecafluoro-1,1,2,2-tetrahydrooctyl-1-trichlorosilane, Gelest) for $30 \mathrm{~min}$, and microstamps were obtained by molding the silanized silicon master with polydimethylsiloxane (PDMS) (Sylgard 184 Silicone Elastomer Kit; Dow Corning, Midland, MI) cured for $4 \mathrm{~h}$ at $60^{\circ} \mathrm{C}$. PDMS stamps were oxidized in an ultraviolet $/ \mathrm{O}_{3}$ oven for $7 \mathrm{~min}$ and inked with a FN solution $(18 \mathrm{mg} / \mathrm{ml}$ ) from human plasma (Sigma, St Louis, MO) for $1 \mathrm{~h}$ at room temperature. Then PDMS stamps were dried under filtered air and gently 
pressed onto a flat PDMS-coated glass coverslip. Uncoated regions were passivated by incubating the glass substrates for $5 \mathrm{~min}$ in a 1\% Pluronic F-127 solution (BASF, Mount Olive, NJ) and washed three times in $\mathrm{PBS}^{26,27}$. The error bars on the microstripe width correspond to the experimental variability of the microstripe width. The experimental variability on the microstripe width mainly depends on the quality of the microstructured PDMS stamp and also on the applied pressure during the microprinting process. The mean value of each microstripe width and its associated standard deviation was obtained by measuring the width of each FN microstripe in epifluorescent mode (12 $\leq \mathrm{n} \leq 16$ per width). The Primo method based on a Light Induced Molecular Adsorption of Proteins (LIMAP) technology (Alveole Company, France) was used to create a pattern of FN composed of five interconnected microstripes of increasing widths (from 5 to $21 \mu \mathrm{m}$, with an increment of 4 $\mu \mathrm{m})$. The 5, 9, 13 and $17 \mu \mathrm{m}$ wide segments each had a length of $60 \mu \mathrm{m}$, whereas the $21 \mu \mathrm{m}$ wide segment was $80 \mu \mathrm{m}$ long.

Preparation of polydimethylsiloxane elastomers. Polydimethylsiloxane (PDMS) substrates of $3 \mathrm{MPa}$ were prepared from the commercially available Sylgard 184 silicone elastomer kit (Dow Corning, Midland, MI) by mixing the base and the curing agent as 10:1 w/w ratio ${ }^{28}$. Pre-polymer solutions were mixed thoroughly for at least $5 \mathrm{~min}$, degassed, and spin-coated at $5000 \mathrm{rpm}$ on $25 \mathrm{~mm}$ glass coverslips ${ }^{29}$. PDMS was then cured for 3 hours at $60^{\circ} \mathrm{C}$. Samples were stored at room temperature in a vacuum desiccator.

Cell culture. Keratocytes are harvested from scale of Central American cichlid Hypsophrys Nicaraguensis. A Fish scale was putted onto a glass coverslip of $25 \mathrm{~mm}$ previously washed in a $30 \%$ ethanol solution and dried. The scale was sandwiched between two $25 \mathrm{~mm}$ diameter glass coverslips and cultured in Leibovitz's Media (L-15) supplemented with 10\% FBS, $1 \%$ antibiotic-antimycotic, $14.2 \mathrm{mM}$ HEPES and 30\% deionized water at room temperature for 12 hours ${ }^{18}$. Individual keratocytes were then dissociated by incubating the tissue in a trypsin solution ( $1 \mathrm{ml}$ per glass slide) for $5 \mathrm{~min}$ and resuspended in $4 \mathrm{ml}$ of L-15 Leibovitz complete medium. Suspended cells were then deposited on microprinted coverslips to investigate their 
migration behaviour. All experiments were made between 2 and 8 hours after cell seeding. To ensure that stationary cells were alive, only cells that actively probed their microenvironment by generating dynamic lamellipodial protrusions over the course of the experiment $(\sim 1800$ sec) were used.

Immunocytochemistry. Intracellular components were made visible using fluorescent staining techniques. Fish keratocytes were rinsed 3 times with PBS (pH 7.4), fixed and permeabilized with a first 1 min incubation in $0.05 \%$ glutaraldehyde and $0.1 \%$ triton $\mathrm{X}-100$ followed by a first rinse in PBS then a second 10 min incubation in a $0.2 \%$ solution of glutaraldehyde. For vinculin immunostaining, cells were permeabilized and fixed with a solution of $4 \%$ paraformaldehyde (Electron Microscopy Sciences, PA) and $0.05 \%$ Triton X100 (Sigma) in PBS (PAA Laboratories) for 15 min and washed three times in PBS. After permeabilization and fixation, cells were blocked with a solution of 5\% FBS. Coverslips were rinsed extensively with PBS and then incubated for 45 min at $37^{\circ} \mathrm{C}$ with Alexa Fluor 488 phalloidin (Invitrogen, 1:200) for staining filamentous actin, DAPI (Invitrogen, 1:200) to visualize the nuclei. To visualize vinculin, the samples were first incubated a primary antibody (anti-vinculin antibody produced in mouse, Sigma-Aldrich, HVIN-1 clone, 1:200) or anti-tubulin antibody produced in mouse (1:200) then tetramethylrhodamine-labelled secondary antibody (goat anti-mouse 1:200, Sigma-Aldrich) was used for $45 \mathrm{~min}$ at $37^{\circ} \mathrm{C}$. Slides were mounted in Slow Fade Gold Antifade (Molecular Probes, Invitrogen).

Epifluorescence and confocal microscopy imaging. Fixed and immunofluorescence-stained preparations were observed in epifluorescence and confocal mode with a Nikon Eclipse Ti-E motorized inverted microscope (Nikon C1 scanhead; Nikon, Japan) equipped with $\times 40$ DIC, ×40 Plan Apo (NA 1.45, oil immersion), ×60 Plan Apo (NA 1.45, oil immersion) and ×100 Plan Apo (NA 1.45, oil immersion) objectives, two lasers (Ar-ion 488 nm; HeNe, 543 nm) and a modulable diode (408 nm). Epifluorescence images were recorded with a Roper QuantEM:512SC EMCCD camera (Photometrics, Tucson, AZ) using NIS Elements Advanced Research 4.0 software (Nikon) (Photometrics, Tucson, AZ) using Capture-Pro so 
ware (Photometrics). Confocal images were acquired with NIS Elements Advanced Research 4.0 software (Nikon) by using small Z-depth increments between focal sections $(0.15 \mu \mathrm{m})$.

Cell tracking. Time-lapse microscopy experiments were done to follow the keratocyte migration. These measurements were performed in DIC mode with a Nikon Eclipse Ti-E motorized inverted microscope at $\times 40$ magnification. Tracking of the keratocytes and kymographs were done with NIS Elements Advanced Research 4.0 software (Nikon, Japan) and analyzed with Origin 8.5 (OriginLab, Northampton, MA). Selected cells were highlighted and their centroids at each time point were recorded ${ }^{18}$. A cell was considered as "stationary" when its center of mass did not move over a period of $1800 \mathrm{sec}$. Stationary cells possessed two lamellipodia at opposite sides of their cell body, which was localized at the center of the cell. For each microstripe width, the migration velocity was determined from the evolution of the distance as a function of time. Distance versus time curves were fitted with a linear regression to determine the slope that corresponds to the mean migration velocity. Then the average of slopes (from 12 to 16 cells, pooled from 3 different experiments) was taken to determine the migration velocity on a specific microstripe width.

Traction force microscopy data measurement and analysis. Hydroxy-polyacrylamide (hydroxy-PAAm) hydrogels ${ }^{30}$ containing 1:50 volume of carboxylate- modified fluorescence latex beads (0.2 $\mu \mathrm{m}$ Fluospheres, Molecular Probes, Eugene, OR) were fabricated on 25-mm diameter glass coverslips according to a protocol previously described ${ }^{31}$. The Young's modulus of the gel was estimated to be $12 \mathrm{kPa}$ using Dynamic Mechanical Analysis (Mettler Toledo, Switzerland). FN-coated PDMS stamps were allowed to react with the activated gel for $1 \mathrm{~h}$ at room temperature to create FN-coated adhesive islands on the gel surface. After removal of the excess of FN by washing with PBS, unprinted areas of the gel were passivated with a bovine serum albumin (BSA) solution overnight. Fluorescence images of the gel containing fluorescent beads immediately beneath the patterned cells were taken during the cell migration and the bead displacements were measured in Matlab between consecutive frames. The traction field was then calculated from the displacement field by solving the 
inverse of the Boussinesq solution from the displacement field on the surface of an elastic halfspace to obtain the traction field when the mechanical properties of the gel are known ${ }^{32}$. The interior of the cell was subdivided into $64 \mu \mathrm{m}^{2}$ squares to approximate the discretized localization of contractile forces.

Determination of the lamellipodial protrusive forces. An atomic force microscope (AFM) cantilever (Bruker, SNL-10) was glued in the vertical position to a glass micropipette, which was controlled by a motorized 3-axis micromanipulator. The AFM cantilever of calibrated spring constant was placed vertically perpendicular to the substrate surface, in front of the leading edge of an individual keratocyte migrating on a FN-coated microstripe. The load force applied by a migrating cell on the advancing leading edge exerts in response an equal and opposite protrusive force to the cantilever. The spring constant of the cantilever was measured according to the thermal noise method $^{33}$ and was found to be $\sim 0.06 \mathrm{~N} / \mathrm{m}$. The load force applied by a migrating cell on the advancing leading edge exerts in response an equal and opposite protrusive force to the cantilever. The vertical position of the cantilever was measured optically using a high magnification objective, from initial contact with the leading edge at $\mathrm{t}=0$ to the deflection of the cantilever at $\mathrm{t}+\partial \mathrm{t}$. The cantilever deflection increased with time until a stall force was reached. Protrusive forces were then estimated using the Hook's law $F=k . x$, where $\mathrm{F}$ is the force $(\mathrm{N}), k$ the spring constant of the AFM cantilever $(\mathrm{N} / \mathrm{m})$ and $x$ the deflection of the cantilever $(\mu \mathrm{m})$.

AFM profile. Cells confined on FN microstripes of 5, 10 and $20 \mu \mathrm{m}$ wide were permeabilized in a solution of $0.05 \%$ glutaraldehyde and $0.1 \%$ Triton X-100 in PBS for 1 min then fixed in a solution of $0.2 \%$ glutaraldehyde in PBS for 10 minutes. Fixed cells were finally dehydrated with water/alcohol solutions of increasing ethanol ratios $(10 \%, 20 \%, 50 \%$, $70 \%$ and $100 \%$ of ethanol). Dehydrated samples were mounted on an ICON Dimension Atomic Force Microscope (Bruker Company) working in Peak Force Quantitative Nanomechanical Mapping (QNM) mode. AFM profiles were acquired on dried cells using calibrated SNL-10 (Bruker) cantilevers. We used a scan rate of $0.2 \mathrm{~Hz}$ and a scan size ranging 
from $5 \times 5 \mu \mathrm{m}^{2}$ to $40 \times 40 \mu \mathrm{m}^{2}$ to acquire AFM profiles perpendicular to the long cell axis. We impose a typical thickness interval for each AFM profile of $2 \mu \mathrm{m}$ and 8 to 12 AFM profiles were acquired per cell $(n=8)$ to cover the whole cell length, depending on the confinement condition (see Rebuttal Figure 5). Finally, each AFM profile was integrated and the mean cellular volume was estimated by summing all profile areas multiplied by profile interval for cells confined on FN microstripes of 5, 10 and $20 \mu$ m wide ( $n=8$ for each conditions, Supplementary Fig. 5).

F-actin live imaging. Fish keratocytes were incubated in a solution of SirActin at $80 \mathrm{nM}$ for $1 \mathrm{~h}$ in medium Leibovitz-15. Then, the medium was changed with a solution of $20 \mu \mathrm{M}$ of Verapamil in medium. SirActin-labeled cells were visualized with a Cy5 cube. The concentration of the probe in the media was kept constant at $80 \mathrm{nM}$ during the whole experiment to get a constant signal. The image correction tool introduced by T. Peng and coworkers ${ }^{34}$ has been used to improve the intensity quantification of Sir-actin time-lapse experiments. This method corrects the intensity profile of each frame and removes both spatial shading effects and the temporal drift.

Statistical analysis. Differences in means between groups were evaluated by two-tailed Student's t-tests performed in Origin 8.5 (OriginLab, Northampton, MA). For multiple comparisons the differences were determined by using an analysis of variance (ANOVA) followed by Tukey post-hoc test. ${ }^{*} \mathrm{p}<0.05,{ }^{*} \mathrm{p}<0.01,{ }^{* *} \mathrm{p}<0.001$ and n.s. is not significant. Unless otherwise stated, all data are presented as mean \pm standard deviation (s.d.).

\section{Data availability}

The data that support the plots within this paper and other findings of this study are available from the corresponding authors upon request. 



\section{A}

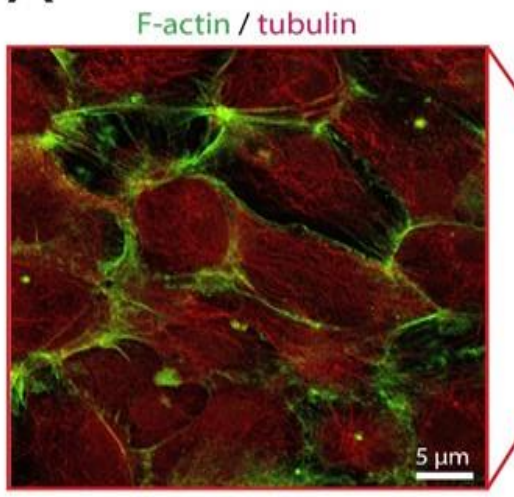

followers

B
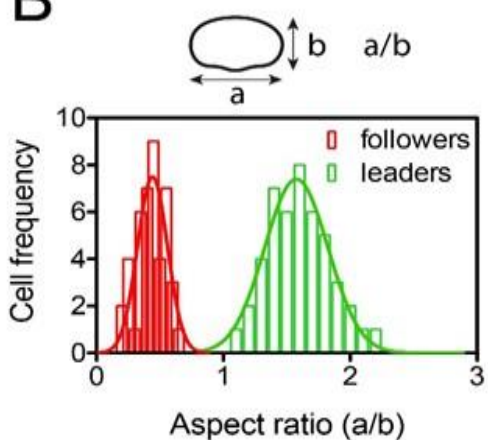

E

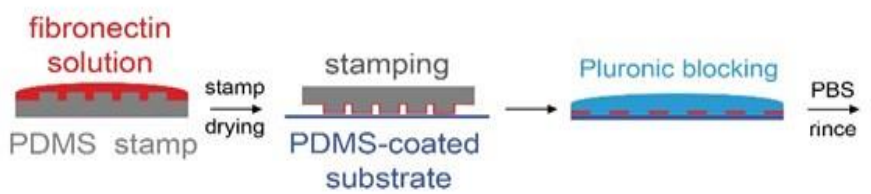

$\mathrm{F}$

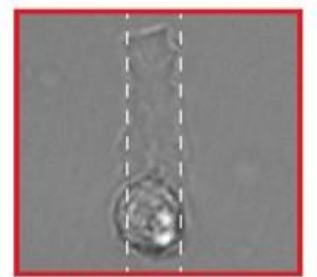

$\mathrm{w}=5 \mu \mathrm{m}$
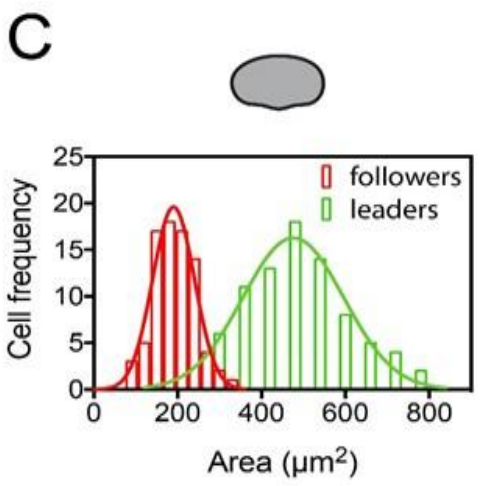

epithelial tissue
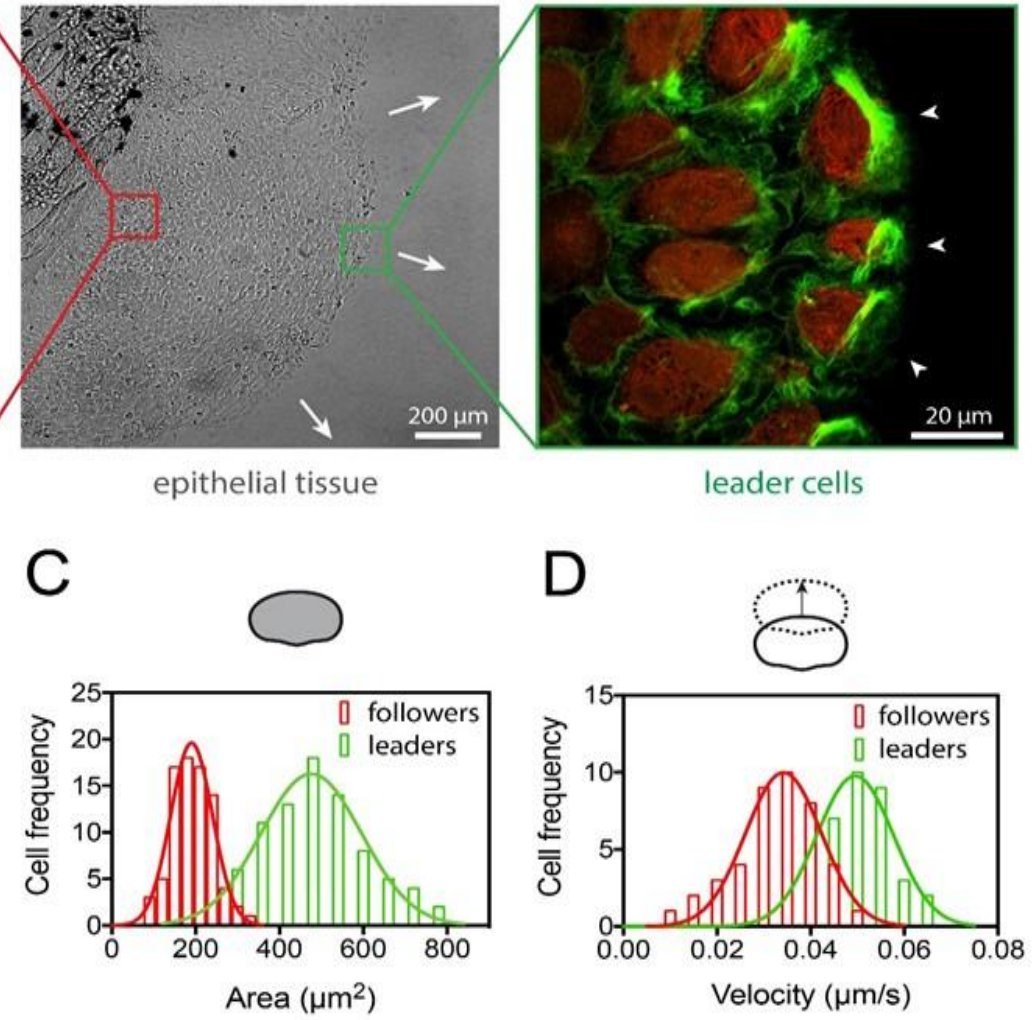

\section{micropatterned} PDMS substrate

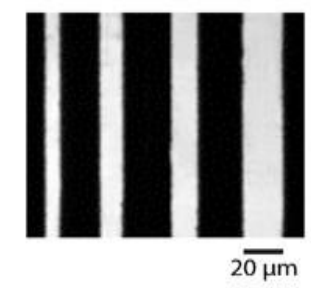

$2 \overline{0 \mu \mathrm{m}}$

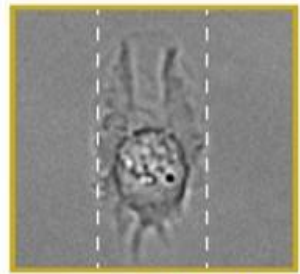

$\mathrm{w}=10 \mu \mathrm{m}$

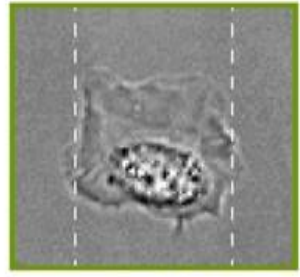

$\mathrm{w}=15 \mu \mathrm{m}$

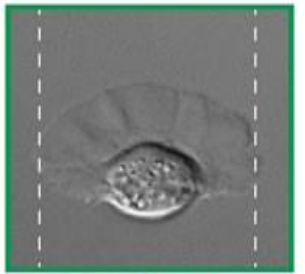

$w=20 \mu m$

Lateral confinement

follower-like

leader-like

Figure 1- Epithelial cells are confined within epithelial tissues. (A) Growing epithelial tissue is composed of confined (left) and leader (right) cells. Confined (in red) and leader (in green) epithelial cells are characterized by different (B) aspect ratios, $(\mathrm{C})$ projected areas and (D) velocities. (E) A microcontact printing technique was used to create fibronectin-coated 
microstripe of different widths distributed in a non-adhesive background. (F) DIC images of individual migrating keratocytes confined on adhesive tracks of different widths ranging from $5 \mu \mathrm{m}$ to $20 \mu \mathrm{m}$. Microstripes reproduce the variability of cell morphologies observed in epithelial tissues due to lateral confinement induced by the proximity of other cells. 
A

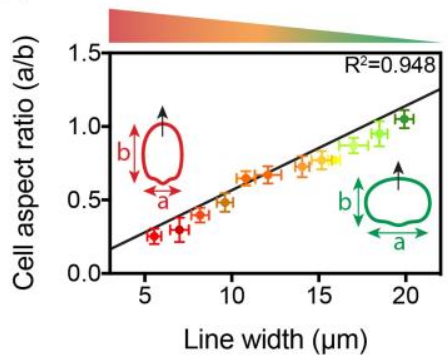

D

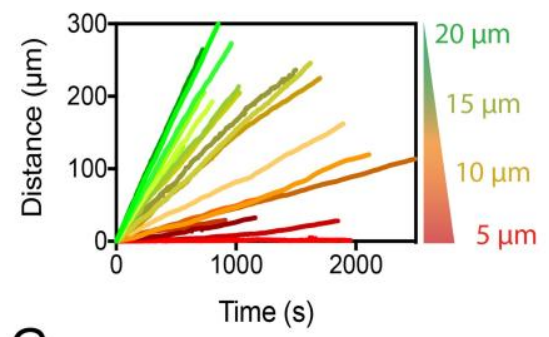

G

\section{Lateral confinement}
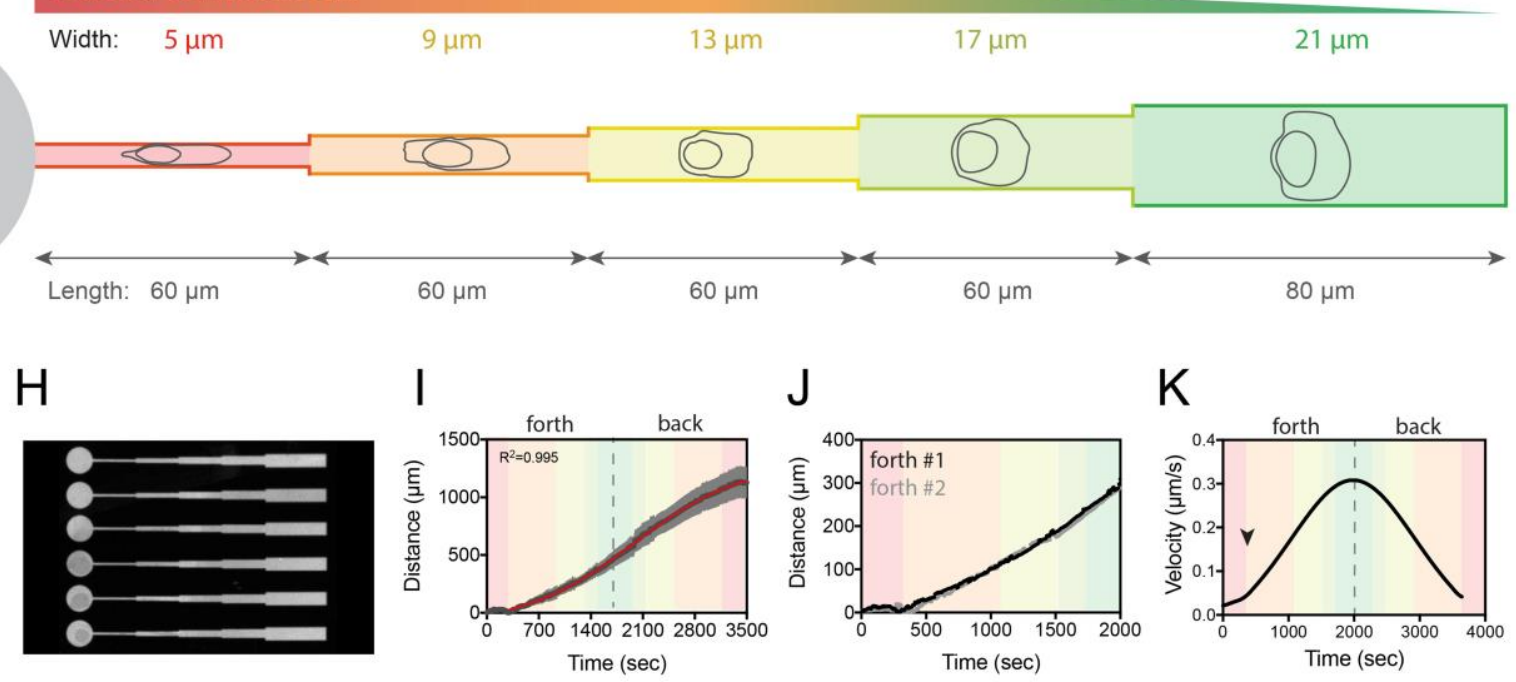

Figure 2- Cell morphologies and migrating velocities are regulated by the lateral

confinement. (A) The cell aspect ratio changes linearly with the width of the micropatterned adhesive microstripe. Errors bars indicate s.d. with $12 \leq \mathrm{n} \leq 16$ for each point. Black line is a linear fit with $\mathrm{R}^{2}=0.948$. (B) The fraction of stationary cells is modulated by the microstripe width. Errors bars indicate s.d. with $n=48$ for each bar. (C) Evolution of the projected area of the leading edge (in black), the whole cell (in light grey), and the cell body (in dark grey) as a function of the microstripe width. Errors bars indicate s.d. with $12 \leq \mathrm{n} \leq 16$ for each point, pooled from three different experiments. (D) Temporal evolution of the migration distance on 
varying microstripe widths represented in different shades of the reference colour. Variation of the migrating velocity as a function of $(\mathrm{E})$ the microstripe width and $(\mathrm{F})$ the cell projected area. Red and green lines are linear regression for low-migrating and fast-migrating cells, respectively. Errors bars for (E) and (F) indicate s.d with $12 \leq \mathrm{n} \leq 16$ cells, pooled from three different experiments. (G) Schematic representation of a micropattern composed of five interconnected stripes of different widths: 5, 9, 13, 17 and $21 \mu \mathrm{m}$. Each segment has a length of $80 \mu \mathrm{m}$ and the total length of this micropattern with variable width is $400 \mu \mathrm{m}$. The narrowest segment is connected to a circular disk of $50 \mu \mathrm{m}$ in diameter. The width of each segment is colour-coded from red (narrower segment: confined state) to green (larger segment: unconfined state). (H) Fluorescence image of six micropatterns of FN deposited on a PDMS coated coverslip. (I) Non-linear evolution of the distance over time during a forth and back travel. Errors bars in light grey indicate s.d with $n=13$. The red curve is a sigmoidal fit with $\mathrm{R}^{2}=0.995$. (J) Superimposed distance curves for two successive travels along the micropattern by the same cell. (K) Evolution of the migrating velocity over time obtained from (I). The arrow shows the transition in cell velocity and the colour of the stripes superimposed on the curve corresponds to the width of each segment: $5 \mu \mathrm{m}$ in red, $9 \mu \mathrm{m}$ in orange, $13 \mu \mathrm{m}$ in yellow, $17 \mu \mathrm{m}$ in light green and $21 \mu \mathrm{m}$ in green. 


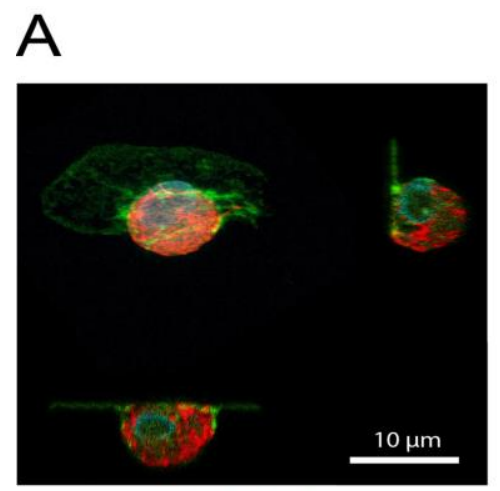

isolated

$\mathrm{D}$

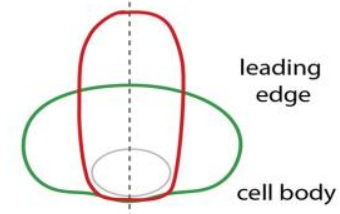

G

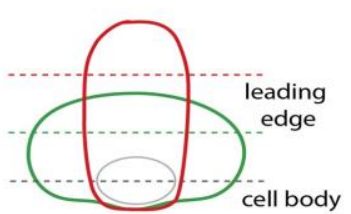

ell body

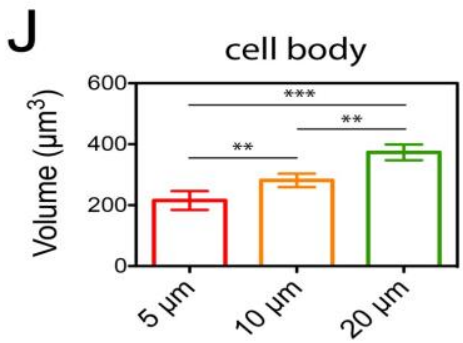

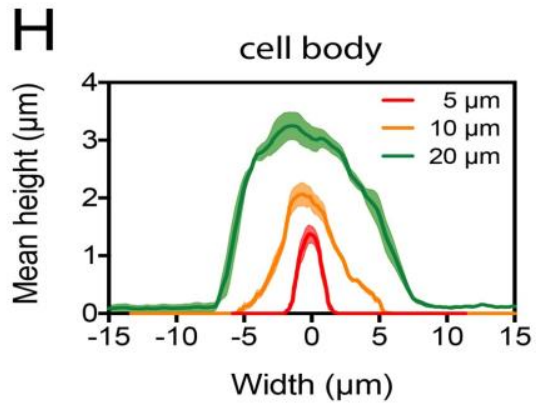

B

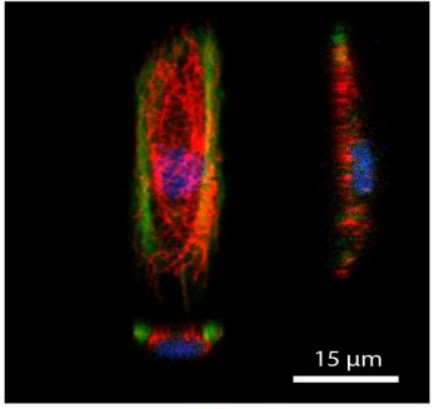

confined

E

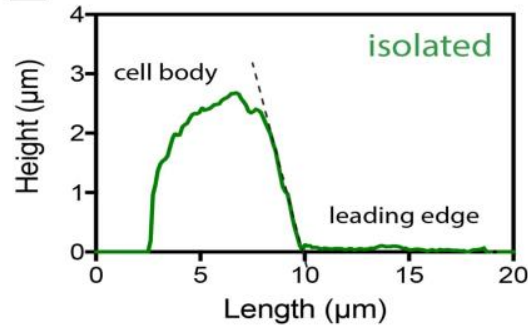

$\mathrm{K}$

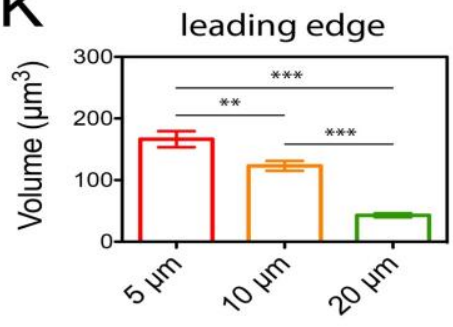

C

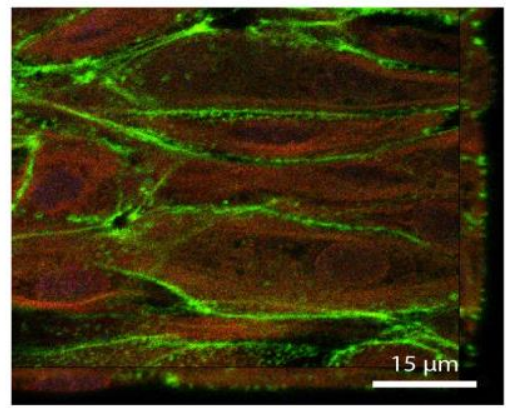

epithelial tissue

$\mathrm{F}$

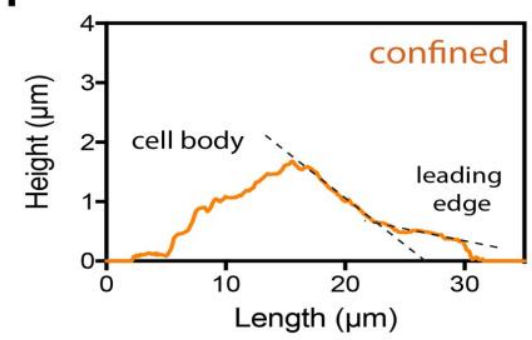

I

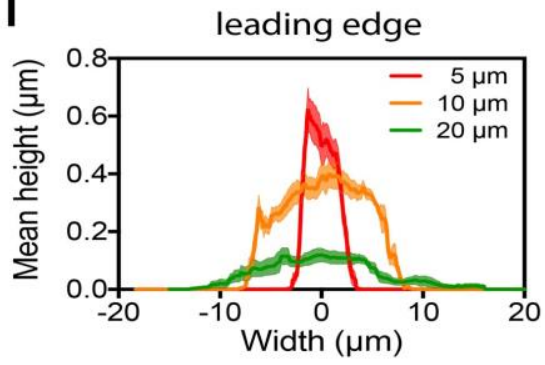

L

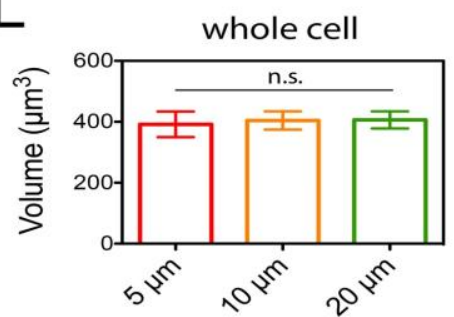

Figure 3- 2D confinement modulates the 3D cellular morphology. Orthogonal views acquired by confocal microscopy of individual keratocytes migrating on (A) a homogeneous FN-coated substrate and (B) a FN microstripe of $15 \mu \mathrm{m}$ wide. (C) Orthogonal confocal view of an epithelial tissue. (D) AFM was used to acquire cell profiles along a line running from the cell rear to the cell front at the cell midline (dotted line) for isolated and confined cells. (E) and (F) shows representative height profiles measured for an isolated cell and a confined cell, respectively. (G) Schematic representation showing the position of the lateral AFM 
height profiles (dotted lines) recorded for $(\mathrm{H})$ the cell body $(\mathrm{n}=11)$ and $(\mathrm{I})$ the leading edge $(\mathrm{n}=13)$ of individual keratocytes migrating on different microstripe widths ( $\mathrm{w}=5 \mu \mathrm{m}$ in red, 10 $\mu \mathrm{m}$ in orange and $20 \mu \mathrm{m}$ in green). Errors bars indicate s.d. The volumes of ( $\mathrm{J})$ the cell body, (K) the leading edge and (L) the whole cell were estimated from AFM profiles and optical images of the cell morphology. Errors bars in $(\mathrm{J}),(\mathrm{K})$ and $(\mathrm{L})$ indicate $\mathrm{s.d}$ for $8 \leq \mathrm{n} \leq 12$ AFM profiles with $\mathrm{n}=8$ cells. ${ }^{* *} \mathrm{p}<0.01, * * * \mathrm{p}<0.001$ and $\mathrm{n} . \mathrm{s}$. is not significant. 
A

Side view

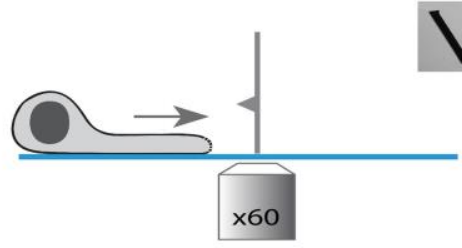

$\mathrm{t}=\mathbf{0}$

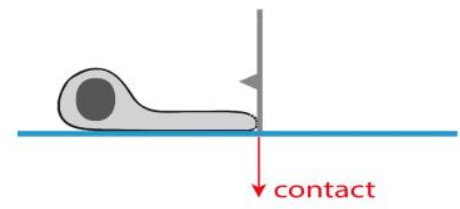

$t_{0}+\partial t$
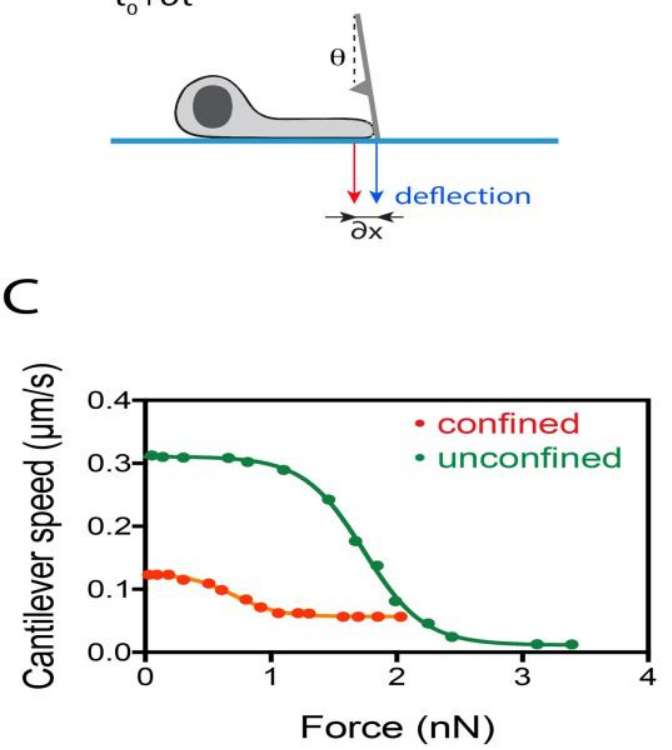

$\mathrm{E}$

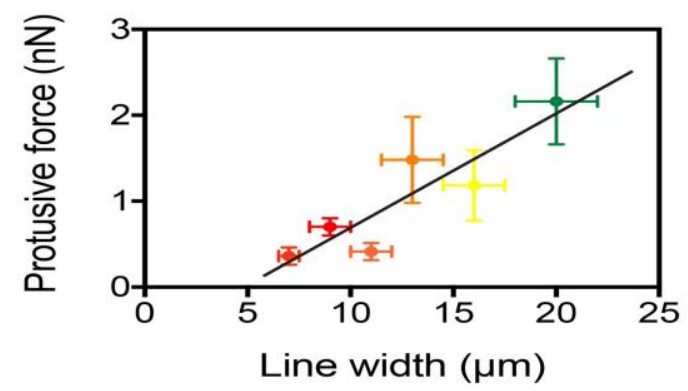

B

Top view
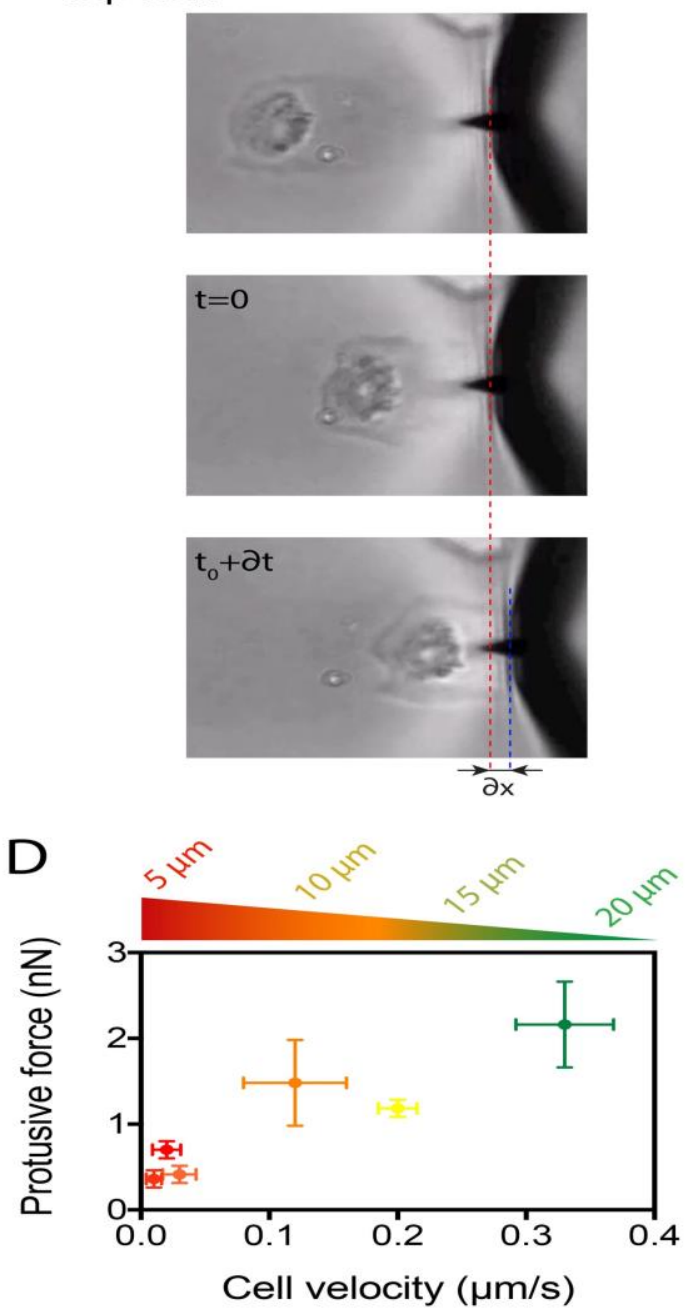

$\mathrm{F}$

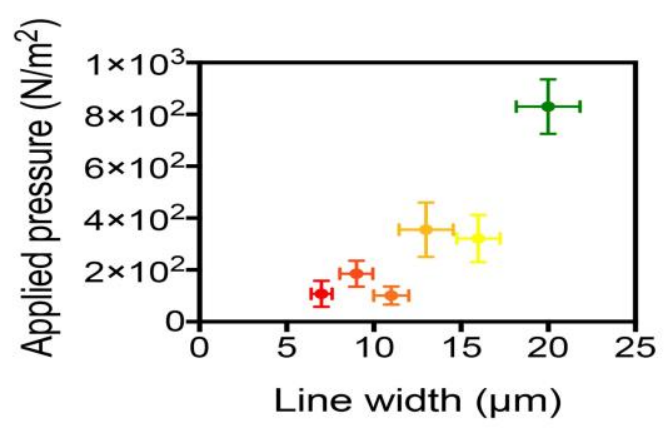

Figure 4- Thicker lamellipodia exert less protrusive forces. The protrusive force exerted by the leading edge decreases with confinement. (A) Schematic representation (side view) of a migrating cell deflecting an AFM cantilever. (B) Top view phase micrographs of the cantilever deflection in response to the pushing force exerted by the leading edge of the oncoming cell. The dashed red line represents the initial position of the cantilever and the dashed blue line shows the cantilever deflection. Contact between the cell and the cantilever 
occurs at $\mathrm{t}=0 \mathrm{~s}$. (C) Typical curves of the cantilever deflection versus time for a confined cell (in red) and an unconfined cell (in green). (D) Protrusive force plotted as a function of cell velocity. (E) The protrusive force and (F) and the applied pressure decrease with the lateral confinement. Errors bars in (D), (E) and (F) indicate s.d for $11 \leq \mathrm{n} \leq 18$ cells. 
A

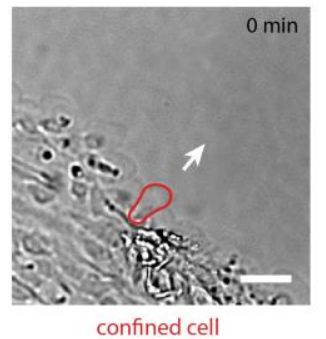

B

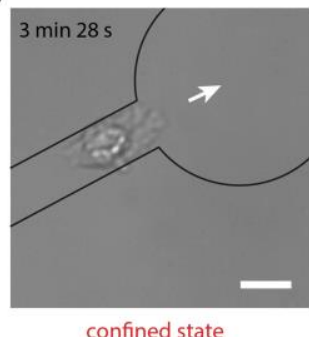

$\mathrm{F}$

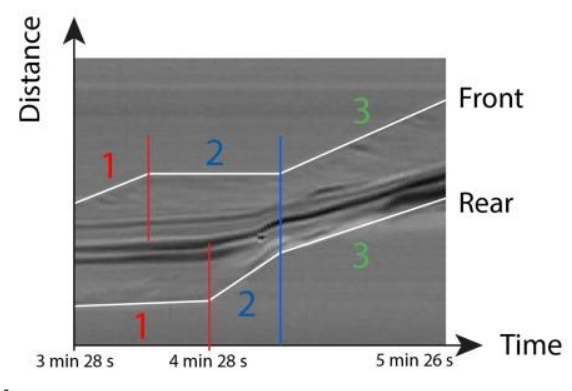

|

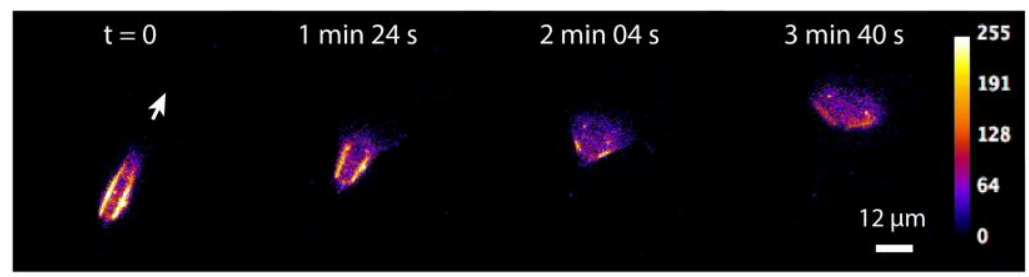

C

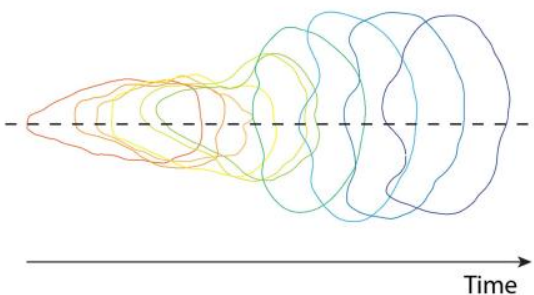

D

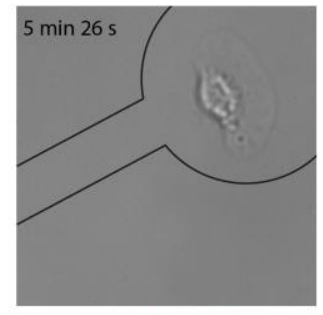

unconfined state

G

leading edge

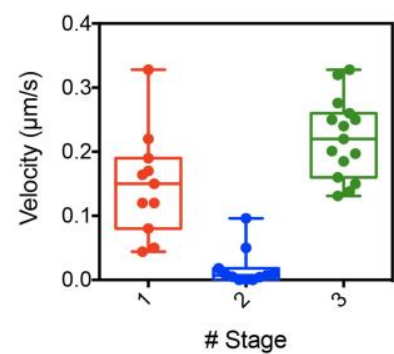

$\mathrm{E}$ micropattern
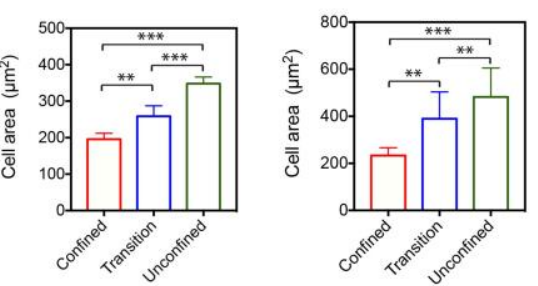

$\mathrm{H}$ trailing edge
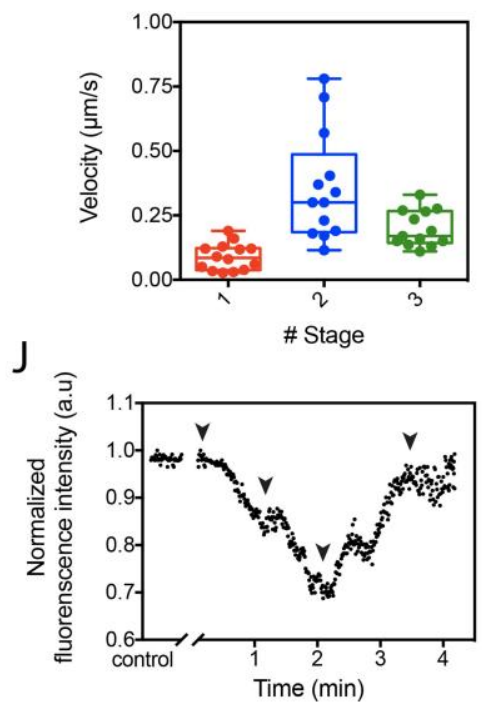

Figure 5- Escaping a tissue requires the reorganization of the actin cytoskeleton. (A)

Time series showing the three main stages allowing a leader cell to escape from an epithelial tissue. (B) The escape process was reproduced by using micropatterns composed of adhesive microstripes of $12 \mu \mathrm{m}$ wide connected to a circular disk of $50 \mu \mathrm{m}$ in diameter. Scale bars are $30 \mu \mathrm{m}$ and $10 \mu \mathrm{m}$ in (A) and (B), respectively. (C) Representation of the outline of a cell escaping from a micropatterned stripe for different time points. Evolution of the cell area during the 3 different stages (confined, transition and unconfined) for cells escaping from (D) a tissue $(n=8)$ and (E) a $12 \mu$ m wide micropatterned stripe $(n=12)$. Errors bars indicate s.d, $* * \mathrm{p}<0.01$ and $* * * \mathrm{p}<0.001$. The dashed line represents the axis used for kymographs. $(\mathrm{F}) \mathrm{A}$ typical kymograph shows three successive stages at the front and at the rear of the cell 
corresponding to the confined stage (in red), the transition (in blue) and the unconfined stage (in green), respectively. Evolution of $(\mathrm{G})$ the front and $(\mathrm{H})$ the rear of a cell escaping from a micropatterned stripe. (I) Sequence showing a SirActin labelled cell escaping from a micropattern. The fluorescence intensity is colour-coded and the scale bar is $12 \mu \mathrm{m}$. (J) Evolution of the normalized actin intensity during the transition from a confined to an unconfined area. $* * \mathrm{p}<0.01$ and $* * * \mathrm{p}<0.001$. 

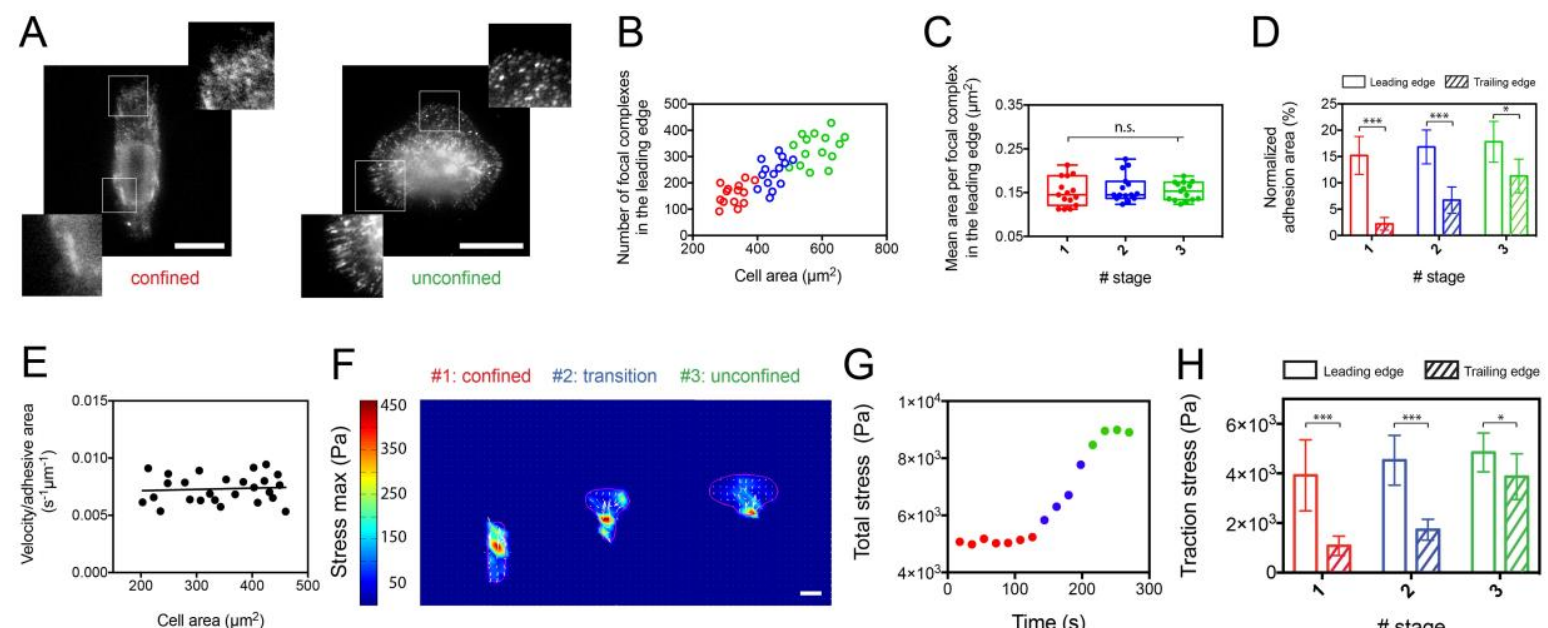

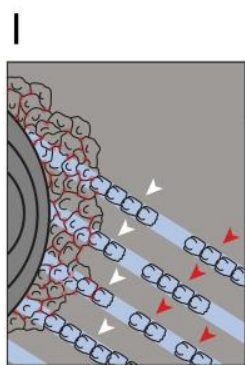

M

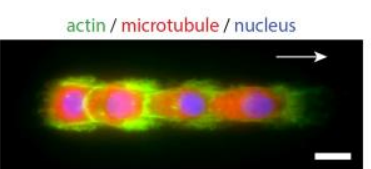

train of cells $(n=4)$

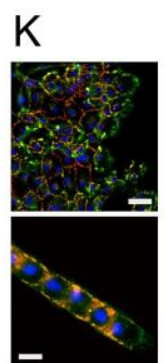

$\mathrm{N}$

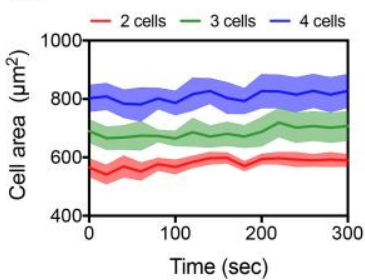

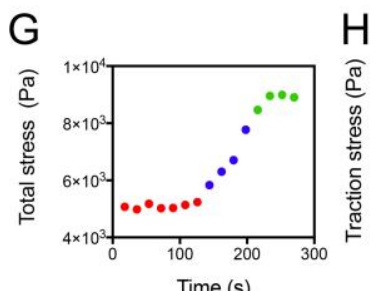

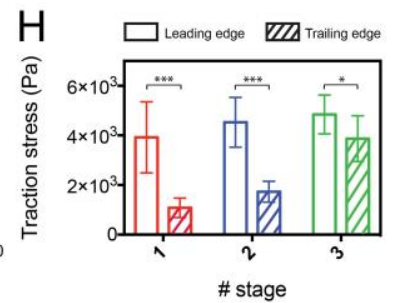

$\mathrm{L}$
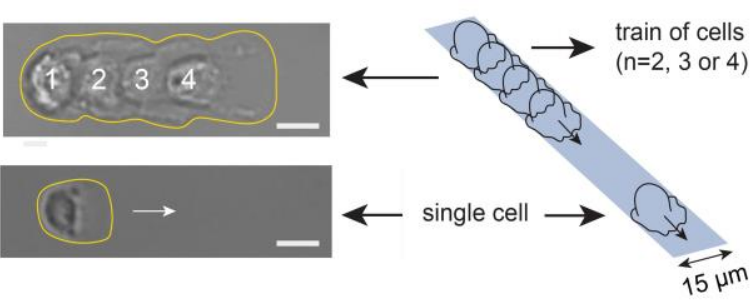

$\mathrm{O}$

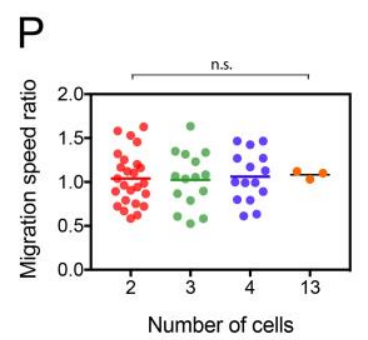

Figure 6- Migration velocity is correlated to cell-substrate adhesive area. (A) Typical

fluorescence images of a confined and unconfined keratocytes stained for vinculin. Zooms indicate the presence of focal complexes in the leading edge and mature focal adhesion in the trailing edges. Scale bars are $12 \mu \mathrm{m}$. (B) Evolution of the number of focal complexes as a function of the cell area for the confined state (in red), the transition (in blue) and the unconfined state (in green). (C) Mean area per focal adhesions for the three successive stages ( $n=16$ for confined, $n=17$ for transition, $n=16$ for unconfined and n.s. is not significant). (D) Normalized adhesion area for the leading (plain bars) and trailing (dashed bars) edges for the three successive stages ( $n=16$ for confined, $n=17$ for transition and $n=16$ for unconfined). Error bars indicate s.d. with $* \mathrm{p}<0.05$ and $* * * \mathrm{p}<0.001$. (E) Normalization of the cell velocity by the adhesive area as a function of the cell spreading area. (F) Sequence of traction force maps during the three main stages (confined, transition and unconfined) of the escape process. The scale bar is $15 \mu \mathrm{m}$. (G) Evolution of the total stress exerted by migrating keratocytes 
increases during the escape process. $(\mathrm{H})$ The individual contribution of the leading and trailing edges in the total stress was decoupled. Error bars indicate s.d. with *p<0.05 and $* * * \mathrm{p}<0.001$. (I) Schematic representation and (J) micrograph in DIC mode of the formation of trains of cells. An epithelial tissue spreads out from the scale deposited on a glass surface (in grey) micropatterned with FN stripes of $15 \mu \mathrm{m}$ wide (in blue). White arrows show the tissue that forms "fingers" on the microstripes, whereas red arrows indicate train of cells which are separated from the main tissue. The scale bar is $50 \mu \mathrm{m}$. (K) Epifluorescence images of an epithelial tissue (top) and a train of cells (bottom) immunostained for vinculin and imaged at the ventral focal plane (in green) and the central focal plane (in red). Nuclei are shown in blue. Scale bars are $20 \mu \mathrm{m}$ (top) and $15 \mu \mathrm{m}$ (bottom). (L) Typical image in DIC mode with a schematic view of a train of cells ( $n=4$, top) and a single cell (bottom) migrating on a microstripe of $15 \mu \mathrm{m}$ wide. Scale bars are $15 \mu \mathrm{m}$. (M) Epifluorescence image of a representative train of cells ( $\mathrm{n}=4$ cells) immunostained for actin (in green), microtubules (in red) and nucleic acids (in blue). The scale bar is $15 \mu \mathrm{m}$. (N) Temporal evolution of the total area of trains of cells composed of 2 cells (in red), 3 cells (in green) and 4 cells (in blue). The mean appears as a solid line with s.d. as a shaded area around it. (O) Total area as a function of cell number for individual cells (in grey) and trains of cells ( 2 cells in red, 3 cells in green and 4 cells in blue) migrating on a $15 \mu \mathrm{m}$ wide microstripe. Error bars indicate s.d. with $\mathrm{n}=9$ for each condition. (P) Migration speed of trains of cells composed of 2 cells (in red, $n=25$ ), 3 cells (in green, $n=17$ ), 4 cells (in blue, $n=18$ ) and 13 cells (in orange, $n=3$ ) normalized by the mean speed of single cells $(9 \leq \mathrm{n} \leq 18)$ migrating on the same microstripe. 
1. Keller, R. Shaping the Vertebrate Body Plan by Polarized Embryonic Cell Movements. Science 298, 1950-1954 (2002).

2. Farooqui, R. \& Fenteany, G. Multiple rows of cells behind an epithelial wound edge extend cryptic lamellipodia to collectively drive cell-sheet movement. J. Cell Sci. 118, 51-63 (2004).

3. Friedl, P. \& Gilmour, D. Collective cell migration in morphogenesis, regeneration and cancer. Nat. Rev. Mol. Cell Biol. 10, 445-457 (2009).

4. Ilina, O. \& Friedl, P. Mechanisms of collective cell migration at a glance. J. Cell Sci. 122, 3203-3208 (2009).

5. Ridley, A. J. Cell Migration: Integrating Signals from Front to Back. Science 302, 1704-1709 (2003).

6. Murrell, M., Oakes, P. W., Lenz, M. \& Gardel, M. L. Forcing cells into shape: the mechanics of actomyosin contractility. Nat. Rev. Mol. Cell Biol. 16, 486-498 (2015).

7. Mayor, R. \& Etienne-Manneville, S. The front and rear of collective cell migration. Nat. Rev. Mol. Cell Biol. 17, 97-109 (2016).

8. Friedl, P. \& Alexander, S. Cancer Invasion and the Microenvironment: Plasticity and Reciprocity. Cell 147, 992-1009 (2011).

9. Wong, I. Y. et al. Collective and individual migration following the epithelialmesenchymal transition. Nat. Mat. 13, 1063-1071 (2014).

10. Vedula, S. R. K. et al. Emerging modes of collective cell migration induced by geometrical constraints. Proc. Natl Acad. Sci. USA 109, 12974-12979 (2012).

11 Abercrombie, M. \& Heaysman, J. E. Observations on the social behaviour of cells in tissue culture. I. Speed of movement of chick heart fibroblasts in relation to their mutual contacts. Exp Cell Res 5, 111-31 (1953)

12. Stoker, M. G. P. \& Rubin, H. Density Dependent Inhibition of Cell Growth in Culture. Nature 215, 171-172 (1967).

13. Angelini, T. E. et al. Glass-like dynamics of collective cell migration. Proc. Natl Acad. Sci. USA 108, 4714-4719 (2011).

14. Garcia, S. et al. Physics of active jamming during collective cellular motion in a monolayer. Proc. Natl Acad. Sci. USA 112, 15314-15319 (2015).

15 Keren, K. et al. Mechanism of shape determination in motile cells Nature 453, 475480 (2008). 
16. Lacayo, C. I. et al. Emergence of Large-Scale Cell Morphology and Movement from Local Actin Filament Growth Dynamics. PLoS Biology 5, e233 (2007).

17. Fournier, M. F., Sauser, R., Ambrosi, D., Meister, J.-J. \& Verkhovsky, A. B. Force transmission in migrating cells. J Cell Biol 188, 287-297 (2010).

18. Riaz, M., Versaevel, M., Mohammed, D., Glinel, K. \& Gabriele, S. Persistence of fanshaped keratocytes is a matrix-rigidity-dependent mechanism that requires $\alpha 5 \beta 1$ integrin engagement. Sci. Rep. 6, 359 (2016).

19. Verkhovsky, A. B. Orientational Order of the Lamellipodial Actin Network as Demonstrated in Living Motile Cells. Mol. Biol. Cell 14, 4667-4675 (2003).

20. Ofer, N., Mogilner, A. \& Keren, K. Actin disassembly clock determines shape and speed of lamellipodial fragments. Proc. Natl Acad. Sci. USA 108, 20394-20399 (2011).

21. Prass, M., Jacobson, K., Mogilner, A. \& Radmacher, M. Direct measurement of the lamellipodial protrusive force in a migrating cell. J Cell Biol 174, 767-772 (2006).

22. Mogilner, A. \& Oster, G. Cell motility driven by actin polymerization. Biophy. J. 71, 3030-3045 (1996).

23. Borm, B., Requardt, R. P., Herzog, V. \& Kirfel, G. Membrane ruffles in cell migration: indicators of inefficient lamellipodia adhesion and compartments of actin filament reorganization. Exp Cell Res 302, 83-95 (2005).

24. Rolli, C. G. et al. 2012 Switchable adhesive substrates: Revealing geometry dependence in collective cell behavior. Biomaterials 33, 2409-2418

25. Trepat, X., Chen, Z. \& Jacobson, K. Cell migration. Compr. Physiol. 2, 2369-2392 (2012)

26. Lantoine, J., Grevesse, T., Villers, A., Delhaye, G., Mestdagh, C., Versaevel, M., Mohammed, D., Bruyère, C., Alaimo, L., Lacour, S.P., Ris L. \& Gabriele, S. Matrix stiffness modulates formation and activity of neuronal networks of controlled architectures. Biomaterials 89, 14-24 (2016).

27. Versaevel, M., Riaz, M., Grevesse, T. \& Gabriele, S. Cell confinement: putting the squeeze on the nucleus. Soft Matter 9, 6665-6676 (2013).

28. Versaevel, M., Grevesse, T., Riaz, M., Lantoine, J. \& Gabriele, S. Micropatterning Hydroxy-PAAm Hydrogels and Sylgard 184 Silicone elastomers with tunable elastic moduli. Methods Cell Biol. 121, 33-48 (2014).

29. Coppée, S., Gabriele, S., Jonas, A.M., Jestin, J. \& Damman, P. Influence of chain interdiffusion between immiscible polymers on dewetting dynamics. Soft Matter 21, 99519955 (2011). 
30. Grevesse, T., Versaevel, M., Circelli, G., Desprez, S. \& Gabriele, S. A simple route to functionalize polyacrylamide hydrogels for the independent tuning of mechanotransduction cues. Lab Chip 13, 777-1 (2013).

31. Grevesse, T., Versaevel, M. \& Gabriele, S. Preparation of hydroxy-PAAm hydrogels for decoupling the effects of mechanotransduction cues. J. Vis. Exp. 90, 1-8 (2014).

32. Versaevel, M., Grevesse, T. \& Gabriele, S. Spatial coordination between cell and nuclear shape within micropatterned endothelial cells. Nat. Comm. 3, 671 (2012).

33. Butt, H. J. \& Jaschke, M. Calculation of thermal noise in atomic force microscopy. Nanotechnology 6, 1-7 (1995).

34. Peng, T., Thorn, K., Schroeder, T., Wang, L., Theis, F.J., Marr, C. \& Navab, N. A $\mathrm{BaSiC}$ tool for background and shading correction of optical microscopy images. Nat Commun. 8, 14836 (2017).

\section{Acknowledgements}

The authors gratefully acknowledge Carl-Philip Heisenberg for kindly providing the GFPUTR zebrafish line, Andrew Kennard for his technical advices for culture of zebrafish embryos, Mathieu Surin for his technical support with AFM measurements. The authors thank Martial Balland, Beth L. Pruitt, Michael Sixt, Daniel Fletcher, Julie Theriot and Jiandong Ding for insightful discussions. This work was conducted with the financial support from the Belgian National Fund for Scientific Research (F.R.S.-FNRS, Crédit de Recherches J009916F) and FEDER Prostem. D.M, J.L., L.A., C.B., E.V. and M.L. are financially supported by FRIA (F.R.S.-FNRS). G.C. is supported by an ERC CoG grant (MolCellTissMech, agreement 647186).

\section{Author contributions}

D.M. and S.G. conceived the project. G.C. and S.G. supervised the project. D.M. and E.V. performed the experiments. C.B. helped with traction force experiments and data analysis. G.D. and O.T. performed PRIMO experiments to create new adhesive micropatterns. K.G. contributed to the silicon wafer microfabrication. M.V., J.L., L.A., C.B., M.L. contributed resources to the project. D.M., G.C. and S.G. analyze data, wrote the main manuscript text 
and prepared figures. All authors contributed to the interpretation of the results, improved the manuscript and figure presentations. 